\title{
A randomized, controlled comparison of macroscopic conjunctival hyperemia in patients treated with bimatoprost $0.01 \%$ or vehicle who were previously controlled on latanoprost
}

\author{
This article was published in the following Dove Press journal: \\ Clinical Ophthalmology \\ 3 December 2010 \\ Number of times this article has been viewed
}

\author{
E Randy Craven' \\ Ching-Chi Liu² \\ Amy Batoosingh ${ }^{2}$ \\ Rhett M Schiffman ${ }^{2}$ \\ Scott MWhitcup ${ }^{2}$ \\ 'Glaucoma Consultants of Colorado, \\ Denver, CO, USA; ${ }^{2}$ Allergan, Inc., \\ Irvine, CA, USA; This work was \\ presented in part at the 19th Annual \\ Meeting of the American Glaucoma \\ Society (AGS); March 5-8, 2009; San \\ Diego, CA, USA
}

\begin{abstract}
Purpose: To evaluate conjunctival hyperemia associated with bimatoprost $0.01 \%$ treatment in patients who replace latanoprost $0.005 \%$ with bimatoprost $0.01 \%$.

Methods: Randomized, double-masked, vehicle-controlled, multicenter study of patients with ocular hypertension or glaucoma whose intraocular pressure (IOP) was adequately controlled on latanoprost monotherapy. At baseline, patients discontinued latanoprost and were randomized to treatment with once-daily bimatoprost $0.01 \%(n=151)$ or vehicle $(n=71)$. The primary endpoint was the peak change in macroscopic hyperemia (conjunctival hyperemia evaluated by gross visual inspection) from baseline to month 1 .

Results: Bimatoprost $0.01 \%$ was noninferior to vehicle in the mean [standard deviation] peak change from baseline macroscopic hyperemia at month $1(0.18[0.46]$ in the bimatoprost $0.01 \%$ group vs 0.02 [0.32] in the vehicle group, $P=0.009)$. The between-group difference was 0.15 (95\% confidence interval [CI]: $0.04,0.26)$, which was within the predefined margin for noninferiority of 0.5 on a hyperemia grading scale of 0 to +3 . There were no statistically significant between-group differences in the percentage of patients with $\mathrm{a} \geq 1$-grade increase in macroscopic hyperemia from baseline. Mean IOP was decreased from baseline $(-0.7$ to $-1.3 \mathrm{~mm} \mathrm{Hg})$ in the bimatoprost $0.01 \%$ group $(P \leq 0.002)$ and was increased from baseline $(+3.3$ to $+3.6 \mathrm{~mm} \mathrm{Hg})$ in the vehicle group $(P<0.001)$ at month 1 . There were no statistically significant betweengroup differences in adverse events.

Conclusions: Bimatoprost $0.01 \%$ was noninferior to vehicle with respect to conjunctival hyperemia in this study population. Replacement of latanoprost with bimatoprost $0.01 \%$ in patients with ocular hypertension or glaucoma can result in additional IOP reduction without clinically important hyperemia.
\end{abstract}

Keywords: conjunctiva, glaucoma, hyperemia, intraocular pressure, prostaglandin analogs, topical drug administration

\section{Introduction}

Glaucoma is an optic neuropathy that is treated by lowering intraocular pressure (IOP). Prostaglandin analogs including latanoprost, bimatoprost, and travoprost are currently the most effective topical medications available for reducing IOP. ${ }^{1}$ These medications, which are well-tolerated, systemically safe, and conveniently dosed once-daily, have become common first-line treatments for glaucoma. ${ }^{2}$

Patient adherence and persistence with medications is frequently an issue in diseases such as glaucoma that are chronic and generally asymptomatic. Factors potentially affecting adherence and persistence with treatment include patient understanding of the
Correspondence: E Randy Craven Glaucoma Consultants of Colorado, 8I0I E. Lowry Boulevard, II0,

Denver, CO, USA

Tel +3037971900

Fax +303347 1341

Email ercraven@glaucdocs.com 
need for treatment and the convenience, cost, and side effects of treatment. ${ }^{3,4}$ The most common side effect of the prostaglandin analogs is conjunctival hyperemia, ${ }^{5}$ which is believed to result from nitric-oxide-mediated vasodilatation in the conjunctiva. ${ }^{6,7}$ Although the redness is reversible and not associated with inflammation, ${ }^{7-9}$ conjunctival hyperemia led to the discontinuation of $1.3 \%$ to $3.4 \%$ of patients treated with bimatoprost $0.03 \%$, latanoprost $0.005 \%$, and travoprost $0.004 \%$ in their respective 6-month pivotal trials. ${ }^{10-12}$ Moreover, patients may be more likely to discontinue treatment due to side effects in clinical practice than in controlled clinical trials. ${ }^{13}$

Meta-analyses of randomized, controlled clinical trials have indicated that bimatoprost $0.03 \%$ reduces IOP more effectively than latanoprost $0.005 \%$, but is associated with a higher incidence of conjunctival hyperemia. ${ }^{14,15}$ Conjunctival hyperemia associated with bimatoprost $0.03 \%$ treatment is typically mild and usually decreases over time during treatment. ${ }^{5,10,16}$ The occurrence of conjunctival hyperemia with bimatoprost has been shown to be related to the dose $\mathrm{e}^{17}$ and the frequency of dosing. ${ }^{8}$

A new formulation of bimatoprost has been developed to maintain the efficacy and improve the tolerability of bimatoprost treatment. In the reformulation, the concentration of bimatoprost was reduced from $0.03 \%$ to $0.01 \%$. A 1 -year, parallel-group clinical study comparing the new bimatoprost $0.01 \%$ formulation with the original bimatoprost $0.03 \%$ formulation reported equivalent efficacy and improved safety with the new formulation. ${ }^{18}$ Bimatoprost $0.01 \%$ was bettertolerated and associated with less conjunctival hyperemia compared with bimatoprost $0.03 \%$.

The purpose of this masked, controlled study was to further evaluate bimatoprost $0.01 \%$ and the conjunctival hyperemia associated with bimatoprost $0.01 \%$ treatment in patients who replace latanoprost with bimatoprost with no washout between treatments. This strategy of replacement of latanoprost with bimatoprost may be done in practice to achieve better IOP control in selected patients. ${ }^{19}$ Our primary hypothesis was that in patients controlled on latanoprost therapy who replace latanoprost with bimatoprost $0.01 \%$, the change in conjunctival hyperemia is no worse than that seen in patients who replace latanoprost with a vehicle. A secondary hypothesis was that bimatoprost $0.01 \%$ would provide a statistically significant reduction in IOP from the latanoprost-treated baseline despite the reduced concentration of bimatoprost.

\section{Material and methods}

This was a randomized, double-masked, vehicle-controlled, multicenter (15 sites), parallel-group comparison study evaluating conjunctival hyperemia associated with bimatoprost $0.01 \%$ treatment. The study was approved by the institutional review board at each site and all patients provided written informed consent.

Patient eligibility was evaluated at a screening visit and confirmed at a baseline visit two days to three weeks later. Patients at least 18 years old, with a diagnosis of ocular hypertension or glaucoma associated with elevated IOP in each eye who, in the opinion of the investigator, were adequately controlled on latanoprost monotherapy and were able to go without IOP-lowering therapy for one month without significant risk, were potentially eligible for the study. Patients were required to have been treated with latanoprost $0.005 \%$ monotherapy in each eye for at least six weeks prior to the baseline visit. Patients were also required to have no more than $5 \mathrm{~mm} \mathrm{Hg}$ of asymmetry of IOP between eyes at baseline and best-corrected visual acuity equivalent to a Snellen acuity of 20/100 or better in each eye. The primary exclusion criteria included: a score of 1 (mild) or greater for any ocular surface finding (including hyperemia) in either eye on macroscopic or on slit-lamp examination at baseline on latanoprost; central corneal thickness $<500$ microns or $>600$ microns in either eye at screening; uncontrolled systemic disease; active ophthalmic disease other than glaucoma that could interfere with the interpretation of the study data; known allergy or sensitivity to the study medication or its components; history of recurrent seasonal allergies within the past two years; history of severe ocular trauma or refractive surgery at any time or laser, intraocular, or filtering surgery within three months prior to baseline; anticipated use of topical ophthalmic medication other than the study medication during the study; and anticipated change in preexisting therapy for systemic disease (eg, systemic betablocker) that could affect IOP. Female patients who were pregnant, nursing, or of childbearing potential and not using reliable contraception were excluded from the study.

At the baseline study visit, patients discontinued latanoprost therapy and were randomized in a $2: 1$ ratio to treatment with bimatoprost $0.01 \%$ or its vehicle administered in each eye, once-daily, in the evening for one month. The randomization sequence was computer-generated and the randomization code was retained by the study sponsor and made available to the investigators only after the study had ended. The study medications were provided in identically masked bottles and the first dose was administered in the evening of the day of the baseline study visit.

Macroscopic hyperemia, defined as bulbar conjunctival hyperemia apparent on visual inspection, was evaluated by the investigator. A standard 5-point scoring system, based 
on photographic standards for comparison, was used with the following descriptors: 0 (none) = normal, bulbar conjunctival vessels easily observed; +0.5 (trace) $=$ trace flush, reddish-pink color; +1 (mild) $=$ mild flush, reddish color; $+2($ moderate $)=$ bright red color; and +3 (severe $)=$ deep bright, diffuse redness. Macroscopic hyperemia data were collected at $8 \mathrm{AM}, 12 \mathrm{PM}$, and $4 \mathrm{PM}$ at the baseline visit and at a follow-up visit at month 1 . The primary study endpoint was the peak change in macroscopic hyperemia from baseline to month 1 , calculated for each eye by subtracting the largest score across the hourly measurements at baseline (the peak severity score at baseline) from the largest score across the hourly measurements at month 1 (the peak severity score at month 1). For each patient, the eye with the larger peak change in macroscopic hyperemia was used for analysis.

Secondary endpoints included the percentage of patients with $\mathrm{a} \geq 1$-unit increase in macroscopic hyperemia in either eye at each time point at month 1 , the mean change from baseline IOP, safety measures, and health outcomes. IOP was measured with a Goldmann applanation tonometer using a 2-person reading method at 8 AM, 12 PM, and 4 PM at the baseline and month 1 study visits. Two measurements were taken for each eye and a third measurement was taken if the difference between the first two measurements was greater than $2 \mathrm{~mm} \mathrm{Hg}$. The mean (of two measurements) or the median (of three measurements) was computed for each eye and the average of both eyes was used for each patient in the analyses.

Safety measures included adverse events, biomicroscopy, ophthalmoscopy, visual acuity, blood pressure, and pulse rate. Adverse events were coded according to the Medical Dictionary for Regulatory Activities (MedDRA) classification scheme. An adverse event was determined by the investigator to be treatment-related if there was a reasonable possibility that it may have been caused by the study medication. Slit-lamp biomicroscopy was performed at $8 \mathrm{AM}$ at baseline and month 1 with findings reported on a scale of $0=$ none, $0.5=$ trace, $1=$ mild, $2=$ moderate, and $3=$ severe .

Patient-reported change in eye appearance (yes or no) and acceptability of treatment were evaluated using a written questionnaire at baseline and month 1. Physician-reported acceptability of treatment was also evaluated using a questionnaire at month 1 .

The primary statistical analyses evaluated the modified intent-to-treat (mITT) population defined as all randomized patients who received at least one dose of study medication and had macroscopic hyperemia evaluated at all three time points at both the baseline and month 1 visits. As a sensitivity analysis, the primary endpoint of macroscopic hyperemia was also evaluated in the per-protocol population of patients with no major protocol violations. Safety analyses evaluated all randomized patients who received at least one dose of study medication and were seen at a postbaseline visit. Nominal data were analyzed using the Pearson chi-square test or Fisher exact test. Macroscopic hyperemia and IOP data were analyzed using analysis of variance models with treatment and site as main effects. The null hypothesis of noninferiority was that the mean peak change from baseline in macroscopic hyperemia for bimatoprost $0.01 \%$ was at least 0.5 grades greater than that for vehicle. Bimatoprost $0.01 \%$ would be noninferior to the vehicle if the upper limit of the $95 \%$ confidence interval (CI) of the between-group difference (bimatoprost $0.01 \%$ minus vehicle) in the peak change from baseline hyperemia at month 1 was less than 0.5 . Within-group changes in IOP were analyzed using paired $t$ tests. Changes in the percentage of patients with diurnal IOP (defined as the average of measurements taken at $8 \mathrm{AM}$, $12 \mathrm{PM}$, and $4 \mathrm{PM}$ ) of less than $18 \mathrm{~mm} \mathrm{Hg}$ were analyzed using the McNemar test. Between-group differences in treatment acceptability were analyzed using the Wilcoxon rank sum test. The alpha level for statistical significance was 0.05 .

A sample size of 192 patients (128 in the bimatoprost $0.01 \%$ group and 64 in the vehicle group) was estimated to have $90 \%$ power to determine noninferiority of bimatoprost to vehicle in the primary endpoint, assuming a common standard deviation (SD) of 1.0 in the peak change from baseline macroscopic hyperemia. Based on an anticipated dropout rate of $10 \%$, the planned enrollment was 216 patients.

\section{Results}

A total of 222 patients (151 in the bimatoprost $0.01 \%$ group and 71 in the vehicle group) at 15 sites were randomized to receive treatment. The study was completed by $95.4 \%$ of patients $(144 / 151)$ randomized to bimatoprost $0.01 \%$ and $93.0 \%$ of patients $(66 / 71)$ randomized to vehicle. The most common reason for early discontinuation from the study was a protocol violation $(2.6 \%$ of bimatoprost $0.01 \%$ patients and $4.2 \%$ of vehicle patients).

The mITT study population included 98.2\% (218/222) of all randomized patients. Baseline characteristics of these patients are listed in Table 1. Most patients were Caucasian and $78 \%$ were diagnosed with glaucoma in both eyes. The only statistically significant difference in baseline characteristics between treatment groups was an approximately 10-micron greater central corneal thickness in the bimatoprost $0.01 \%$ group $(P=0.017)$; this difference was not considered to be clinically meaningful. The duration of latanoprost monotherapy run-in prior to the baseline visit ranged from at least 
Table I Patient characteristics at baseline

\begin{tabular}{|c|c|c|c|}
\hline & $\begin{array}{l}\text { Bimatoprost } \\
0.01 \% \\
(n=147)\end{array}$ & $\begin{array}{l}\text { Vehicle } \\
(n=7 I)\end{array}$ & $\begin{array}{l}\text { Between-group } \\
P \text { value }\end{array}$ \\
\hline $\begin{array}{l}\text { Mean age in } \\
\text { years (SD) }\end{array}$ & $64.2(11.5)$ & $66.3(10.7)$ & 0.201 \\
\hline (range) & $24-89$ & $28-90$ & \\
\hline Sex, n (\%) & & & 0.694 \\
\hline Male & 60 (40.8\%) & 27 (38.0\%) & \\
\hline Female & 87 (59.2\%) & 44 (62.0\%) & \\
\hline Race, n (\%) & & & $0.115^{\mathrm{a}}$ \\
\hline Caucasian & II 10 (74.8\%) & 47 (66.2\%) & \\
\hline Black & $33(22.4 \%)$ & 23 (32.4\%) & \\
\hline Asian & $4(2.7 \%)$ & I (I.4\%) & \\
\hline Diagnosis & & & 0.613 \\
\hline $\begin{array}{l}\text { Ocular } \\
\text { hypertension } \\
\text { (both eyes) }\end{array}$ & 30 (20.4\%) & I3 (I8.3\%) & \\
\hline $\begin{array}{l}\text { Glaucoma } \\
\text { (both eyes) }\end{array}$ & II 4 (77.6\%) & 55 (77.5\%) & \\
\hline $\begin{array}{l}\text { Ocular } \\
\text { hypertension/ } \\
\text { glaucoma }\end{array}$ & $3(2.0 \%)$ & 3 (4.2\%) & \\
\hline $\begin{array}{l}\text { Mean central } \\
\text { corneal thickness } \\
\text { in microns (SD) }\end{array}$ & $555(30)$ & 545 (29) & 0.017 \\
\hline
\end{tabular}

Notes: ap value for black versus nonblack; ' ${ }^{\circ}$ One eye with ocular hypertension and the fellow eye with glaucoma; 'Analysis used the average value from both eyes of each patient.

Abbreviation: SD, standard deviation.

six weeks to more than two years for patients in this population. Patients used latanoprost between 6 PM and 11 PM the night before the baseline visit.

There was no statistically significant difference between treatment groups in the mean peak macroscopic hyperemia severity score at the latanoprost-treated baseline. The mean (SD) peak score at the latanoprost-treated baseline was $0.32(0.28)$ in the bimatoprost $0.01 \%$ group and $0.30(0.29)$ in the vehicle group $(P=0.466)$. At month 1 , the mean (SD) peak hyperemia score was $0.50(0.51)$, or trace, in the bimatoprost $0.01 \%$ group and $0.32(0.35)$ in the vehicle group $(P=0.004)$ (Figure 1).

After one month of bimatoprost or vehicle treatment, the mean (SD) peak change in hyperemia from the latanoprost-treated baseline was $0.18(0.46)$ in the bimatoprost $0.01 \%$ group and $0.02(0.32)$ in the vehicle group. The between-group difference was 0.15 with a $95 \% \mathrm{CI}$ of $0.04,0.26(P=0.009)$. As the upper limit of the $95 \% \mathrm{CI}$ of the between-group difference was within the predefined noninferiority margin of 0.5 , bimatoprost $0.01 \%$ was noninferior to vehicle in the primary study endpoint of peak change from baseline macroscopic hyperemia. Results of the analysis of macroscopic hyperemia in the per-protocol study population, which excluded an additional 23 randomized

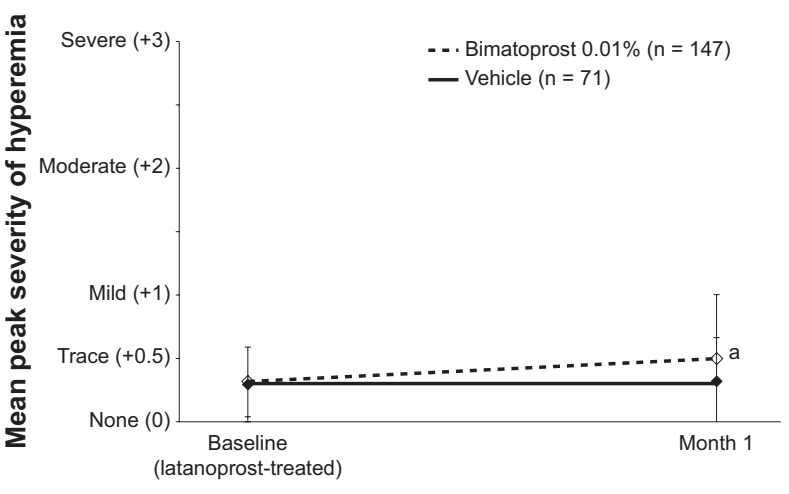

Figure I Mean peak macroscopic hyperemia scores at baseline and month I. Notes: The between-group difference at month I was 0.17 (95\% Cl: $0.06,0.29)$. ${ }^{a} P=0.004$ vs vehicle. Error bars represent the standard deviation.

patients due to protocol violations, were consistent with those in the mITT study population.

There were no statistically significant differences between treatment groups in the percentage of patients demonstrating a $\geq 1$-unit increase in macroscopic hyperemia from baseline at any time point at month 1 . The percentage of patients with a $\geq 1$-unit increase in the severity of macroscopic hyperemia in the bimatoprost $0.01 \%$ and vehicle groups, respectively, was $8.2 \%$ and $1.4 \%$ at $8 \mathrm{AM}$ $(P=0.065), 4.1 \%$ and $1.4 \%$ at $12 \mathrm{PM}(P=0.432)$, and $4.1 \%$ and $0 \%$ at $4 \mathrm{PM}(P=0.181)$.

There were no statistically significant differences in mean IOP between the bimatoprost $0.01 \%$ group and the vehicle group at the latanoprost-treated baseline (Table 2). After one month of treatment with bimatoprost $0.01 \%$ or vehicle, IOP was reduced significantly in the bimatoprost $0.01 \%$ group $(P \leq 0.002)$ and was increased significantly in the vehicle group $(P<0.001)$ at each time point. The mean change from baseline IOP at month 1 ranged from $-0.7 \mathrm{~mm} \mathrm{Hg}$ to $-1.3 \mathrm{~mm} \mathrm{Hg}$ with bimatoprost $0.01 \%$ and from $+3.3 \mathrm{~mm} \mathrm{Hg}$ to $+3.6 \mathrm{~mm} \mathrm{Hg}$ with

Table 2 Efficacy measure: intraocular pressure $(\mathrm{mm} \mathrm{Hg})$

\begin{tabular}{|c|c|c|c|}
\hline & $\begin{array}{l}\text { Bimatoprost } \\
0.01 \% \\
(n=147)\end{array}$ & $\begin{array}{l}\text { Vehicle } \\
(n=7 I)\end{array}$ & $\begin{array}{l}\text { Between-group } \\
P \text { value }\end{array}$ \\
\hline \multicolumn{4}{|c|}{ Mean (SD) baseline IOP on latanoprost } \\
\hline 8 AM & $18.2(3.1)$ & $18.0(3.3)$ & 0.292 \\
\hline $12 \mathrm{PM}$ & $18.0(3.2)$ & $17.9(3.4)$ & 0.626 \\
\hline 4 PM & $17.6(2.9)$ & $17.2(3.6)$ & 0.250 \\
\hline \multicolumn{4}{|c|}{ Mean (SD) change from latanoprost-treated baseline } \\
\hline \multicolumn{4}{|c|}{ IOP at month I } \\
\hline $8 \mathrm{AM}$ & $-1.0(2.6)^{\mathrm{a}}$ & $+3.6(3.9)^{\mathrm{a}}$ & $<0.001$ \\
\hline $12 \mathrm{PM}$ & $-1.3(2.6)^{\mathrm{a}}$ & $+3.3(4.0)^{\mathrm{a}}$ & $<0.001$ \\
\hline 4 PM & $-0.7(2.9)^{\mathrm{a}}$ & $+3.3(3.5)^{\mathrm{a}}$ & $<0.001$ \\
\hline
\end{tabular}

Note: ${ }^{a} P \leq 0.002$ for within-group change from baseline based on paired $t$ test. Abbreviations: SD, standard deviation; IOP, intraocular pressure. 
vehicle (Table 2). In the bimatoprost $0.01 \%$ group, the percentage of patients with diurnal IOP $<18 \mathrm{~mm} \mathrm{Hg}$ increased statistically significantly from $50.3 \%$ at the latanoprost-treated baseline to $66.0 \%$ at one month after replacement of latanoprost with bimatoprost $0.01 \%(P<0.001)$, while in the vehicle group, the percentage of patients with diurnal IOP $<18 \mathrm{~mm} \mathrm{Hg}$ decreased significantly from $56.3 \%$ at the latanoprost-treated baseline to $21.1 \%$ at one month after replacement of latanoprost with vehicle $(P<0.001)$. Notably, among the 73 patients in the bimatoprost $0.01 \%$ group who had diurnal IOP $\geq 18 \mathrm{~mm} \mathrm{Hg}$ at the latanoprost-treated baseline, $38.4 \%$ (28) had diurnal IOP $<18 \mathrm{~mm} \mathrm{Hg}$ after replacement of latanoprost with bimatoprost $0.01 \%$.

There were no statistically significant between-group differences in the proportion of patients with adverse events, treatment-related adverse events, or discontinuations due to adverse events. Adverse events were reported in $20.0 \%$ (30/150) of bimatoprost $0.01 \%$-treated patients and $12.7 \%$ $(9 / 71)$ of vehicle-treated patients. None of the adverse events were serious and only 2 of 150 patients in the bimatoprost $0.01 \%$ group $(1.3 \%)$ discontinued from the study due to adverse events (one patient due to photophobia and one patient due to conjunctival hyperemia, blurred vision, and eye pruritus). The most common treatment-related adverse event in each treatment group was conjunctival hyperemia (Table 3).

On biomicroscopy, there were no statistically significant differences between treatment groups in the percentage of patients with a $\geq 1$-unit increase from the baseline severity of any findings, including conjunctival hyperemia, in either eye (Table 4). In other safety evaluations, there were no significant differences between treatment groups in change

Table 3 Incidence of treatment-related adverse events ${ }^{\mathrm{a}}$

\begin{tabular}{|c|c|c|c|}
\hline Adverse event & $\begin{array}{l}\text { Bimatoprost } \\
0.01 \% \\
(n=150)\end{array}$ & $\begin{array}{l}\text { Vehicle } \\
(n=7 I)\end{array}$ & $\begin{array}{l}\text { Between-group } \\
P \text { value }\end{array}$ \\
\hline Any adverse event ${ }^{b}$ & $15.3 \%(23 / 150)$ & $7.0 \%(5 / 7 \mathrm{I})$ & 0.084 \\
\hline $\begin{array}{l}\text { Conjunctival } \\
\text { hyperemia }\end{array}$ & $6.7 \%(10 / 150)$ & $2.8 \%(2 / 7 \mathrm{I})$ & 0.346 \\
\hline Eye pruritus & $2.7 \%(4 / 150)$ & $\mathrm{I} .4 \%(\mathrm{I} / 7 \mathrm{I})$ & $>0.999$ \\
\hline Dry eye & $2.0 \%(3 / 150)$ & $\mathrm{I} .4 \%(\mathrm{I} / 7 \mathrm{I})$ & $>0.999$ \\
\hline Blurred vision & $1.3 \%(2 / 150)$ & $\mathrm{I} .4 \%(\mathrm{I} / 7 \mathrm{I})$ & $>0.999$ \\
\hline $\begin{array}{l}\text { Foreign body } \\
\text { sensation }\end{array}$ & $0.7 \%(\mathrm{I} / \mathrm{I} 50)$ & $\mathrm{I} .4 \%(\mathrm{I} / 7 \mathrm{I})$ & 0.540 \\
\hline Headache & $0.0 \%(0 / 150)$ & $\mathrm{I} .4 \%(\mathrm{I} / 7 \mathrm{I})$ & 0.321 \\
\hline $\begin{array}{l}\text { Keratoconjunctivitis } \\
\text { sicca }\end{array}$ & $0.0 \%(0 / 150)$ & I.4\% (I/7I) & 0.321 \\
\hline Scleral hyperemia & $0.0 \%(0 / 150)$ & $\mathrm{I} .4 \%(\mathrm{I} / 7 \mathrm{I})$ & 0.321 \\
\hline
\end{tabular}

Notes: aAll treatment-related adverse events reported in $\geq 1 \%$ of patients in either treatment group in the safety population are listed; 'Overall incidence of treatmentrelated adverse events (ie, patients with one or more treatment-related adverse events). from baseline visual acuity or ophthalmoscopic findings and there were no clinically meaningful differences between treatment groups in change from baseline blood pressure and pulse rate.

At month 1, treatment acceptability judged by both physicians and patients was higher with bimatoprost $0.01 \%$ than with vehicle $(P<0.001)$. Physicians were very or extremely willing to continue $85.7 \%(126 / 147)$ of the patients in the bimatoprost $0.01 \%$ group on bimatoprost $0.01 \%$, but were very or extremely willing to continue only $39.4 \%(28 / 71)$ of patients in the vehicle group on vehicle, most commonly because IOP was not low enough. Similarly, the percentage of patients who were very or extremely willing to continue on their study medication at the end of the study was higher in the bimatoprost $0.01 \%$ group $(88.4 \%, 130 / 147)$ than in the vehicle group $(52.9 \%, 37 / 70)$. The most common reason for patients being only somewhat willing or unwilling to continue on bimatoprost $0.01 \%$ was listed as "other" (neither "eye redness" nor "IOP not low enough"). Most patients in each treatment group $(95.1 \%, 137 / 147$ in the bimatoprost $0.01 \%$ group and $94.1 \%, 64 / 70$ in the vehicle group) reported no change in the appearance of their eyes since the beginning of the study.

\section{Discussion}

A goal of therapy in glaucoma is to reduce IOP to a low target level while minimizing the side effects of treatment. A previous study demonstrated that efficacy is maintained, but the occurrence and severity of conjunctival hyperemia is reduced with bimatoprost $0.01 \%$ compared with bimatoprost $0.03 \%{ }^{18}$ The present study further demonstrated that in

Table 4 Proportion of patients with a $\geq$ I-unit increase in the severity of biomicroscopy findings ${ }^{a}$

\begin{tabular}{|c|c|c|c|}
\hline Finding & $\begin{array}{l}\text { Bimatoprost } \\
0.01 \% \\
(n=150)\end{array}$ & $\begin{array}{l}\text { Vehicle } \\
(n=7 I)\end{array}$ & $\begin{array}{l}\text { Between-group } \\
P \text { value }\end{array}$ \\
\hline Any finding ${ }^{b}$ & $6.0 \%(9 / 150)$ & $2.8 \%(2 / 7 \mathrm{I})$ & 0.509 \\
\hline Nuclear cataract & I.3\% (2/I50) & $2.8 \%(2 / 7 \mathrm{I})$ & 0.595 \\
\hline $\begin{array}{l}\text { Conjunctival } \\
\text { hyperemia }\end{array}$ & $4.0 \%(6 / 150)$ & $0.0 \%(0 / 7 \mathrm{I})$ & 0.180 \\
\hline $\begin{array}{l}\text { Superficial } \\
\text { punctate } \\
\text { keratopathy }\end{array}$ & $0.7 \%(1 / 150)$ & $0.0 \%(0 / 7 \mathrm{I})$ & $>0.999$ \\
\hline $\begin{array}{l}\text { Eyelid margin } \\
\text { crusting }\end{array}$ & $0.7 \%(I / I 50)$ & $0.0 \%(0 / 7 \mathrm{I})$ & $>0.999$ \\
\hline
\end{tabular}

Notes: ${ }^{a}$ Biomicroscopy findings were graded on a scale of $0=$ none, $0.5=$ trace, $\mathrm{I}=$ mild, 2 = moderate, and 3 = severe. All biomicroscopy findings with at least a $\mathrm{I}$-unit increase in severity from baseline or screening to month I reported in any patient are listed; 'Overall incidence of increased severity of biomicroscopic findings (ie, patients with increased severity of any finding). 
patients controlled on latanoprost who replaced latanoprost monotherapy with bimatoprost $0.01 \%$ monotherapy, the conjunctival hyperemia attributed to bimatoprost $0.01 \%$ was no worse than that seen in patients who replaced latanoprost monotherapy with vehicle. Moreover, bimatoprost $0.01 \%$ statistically significantly reduced the mean IOP from the latanoprost-treated baseline. Bimatoprost $0.01 \%$ was as well-tolerated as the vehicle and was well-accepted by both patients and physicians.

Using a predetermined noninferiority margin of 0.5 , bimatoprost $0.01 \%$ was noninferior to the vehicle in the mean peak change from baseline hyperemia scores at month 1 . The difference between the bimatoprost $0.01 \%$ and vehicle groups in the change from baseline peak hyperemia scores at month 1 was only 0.15 , a difference that we do not consider to be clinically meaningful. Further, there were no statistically significant differences between the bimatoprost $0.01 \%$ group and the vehicle group in the proportion of patients who had at least a 1-grade increase in hyperemia from baseline to month 1 at any time point. Adverse event reports of conjunctival hyperemia were consistent with a low, reduced occurrence of conjunctival hyperemia associated with bimatoprost $0.01 \%$ compared with the original bimatoprost $0.03 \%$ formulation. There was no statistically significant difference in adverse event reports of hyperemia between the bimatoprost $0.01 \%$ group $(6.7 \%)$ and the vehicle group $(2.8 \%)$. In contrast, large studies evaluating bimatoprost $0.03 \%$ have reported conjunctival hyperemia as an unsolicited adverse event in $21 \%$ to $46 \%$ of treated patients. ${ }^{20-22}$ Although the duration of those studies was longer (3 or 6 months), conjunctival hyperemia associated with prostaglandin analog therapy typically has an early onset; in the 1-year pivotal trials of bimatoprost $0.03 \%$, the median time to onset of conjunctival hyperemia was 14 days after the initiation of therapy. ${ }^{8}$ The low proportion of patients $(4.9 \%)$ reporting a change in eye appearance in the present study is also consistent with an improved side effect profile and reduced conjunctival hyperemia with the bimatoprost $0.01 \%$ formulation. Only one patient $(0.7 \%)$ discontinued from bimatoprost $0.01 \%$ treatment due to conjunctival hyperemia.

In the Advanced Glaucoma Intervention Study, visual field loss was minimized in patients who consistently achieved IOP less than $18 \mathrm{~mm} \mathrm{Hg}{ }^{23}$ In the present study, a statistically significant mean decrease in IOP and increased likelihood of achievement of diurnal IOP less than $18 \mathrm{~mm}$ $\mathrm{Hg}$ was observed in patients who replaced latanoprost with bimatoprost $0.01 \%$. These results are consistent with the literature. ${ }^{14,15,18,24}$ Patients who replaced latanoprost with vehicle experienced a significant increase in IOP, but the magnitude of the increase was smaller than might be expected, given that latanoprost has typically been shown to reduce IOP by approximately $30 \%$. $^{1}$ These results could suggest a possible placebo effect or, perhaps more likely, a continued effect of latanoprost at the month 1 study visit, as a previous study has shown that one month may be inadequate for complete washout of the effect of previous latanoprost treatment. $^{25}$

The bimatoprost $0.01 \%$ formulation and the vehicle used in the study are preserved with benzalkonium chloride (BAK), which appears to promote penetration of topically applied medications by disrupting intercellular adhesions in the corneal epithelium, ${ }^{26}$ thus allowing lower concentrations of drugs to be applied to the ocular surface. Although BAK and drug formulations containing BAK have been reported to cause cell or ocular surface damage in cell culture and rabbit model systems, ${ }^{27-33}$ the clinical significance of these findings is unknown. Clinical studies that have evaluated travoprost containing BAK compared with travoprost containing an alternative preservative have shown no improvement in clinical signs or other safety measures with the BAK-free travoprost formulation. ${ }^{34,35}$ Similarly, no improvement in safety or tolerability has been observed with preservative-free tafluprost compared with tafluprost preserved with BAK. ${ }^{36}$ The concentration of BAK in bimatoprost $0.01 \%$ is the same as that in latanoprost $0.005 \%$, which has been successfully used for many years ${ }^{37,38}$ and because the prostaglandin analogs are dosed only once-daily, preservative exposure and accumulation are minimized. In the phase 3 study comparing bimatoprost $0.01 \%$ with the original bimatoprost $0.03 \%$ formulation, bimatoprost $0.01 \%$ demonstrated improved tolerability over 12 months compared with bimatoprost $0.03 \%$ and no patients discontinued from bimatoprost $0.01 \%$ treatment due to adverse events that could be related to corneal toxicity, such as corneal erosions or punctate keratitis. $^{18}$

There are several limitations to the present study. Because the study duration was one month with no interim follow-up visits, hyperemia that might occur after longer-term treatment with bimatoprost $0.01 \%$ could not be evaluated and hyperemia could have occurred and resolved prior to month 1 in some patients. Further, the study population was restricted to patients who were on latanoprost monotherapy and did not have significant hyperemia at screening. The fact that the mean IOP increased significantly by more than $3 \mathrm{~mm} \mathrm{Hg}$ in patients who switched to the vehicle suggests that the study population was responsive to latanoprost. 
However, it is likely that patients who are switched directly from latanoprost to bimatoprost demonstrate less hyperemia than treatment-naive patients. ${ }^{5,39}$ Further studies will be needed to evaluate conjunctival hyperemia associated with bimatoprost $0.01 \%$ use in treatment-naive patients.

\section{Acknowledgments}

The authors thank Kate Ivins, PhD, for medical writing assistance in the preparation of the manuscript. The study was sponsored by Allergan, Inc. (Irvine, CA, USA) and is registered with the trial identifier NCT00538304 at http:// www.clinicaltrials.gov.

\section{Principal investigators}

E Randy Craven, MD (Denver, CO, USA); Douglas G Day, MD (Atlanta, GA, USA); Ronald EP Frenkel, MD (Stuart, FL, USA); Walter I Fried, MD, PhD, SC (Gurnee, IL, USA); Paul James Hartman, MD (Rochester, NY, USA); Anup K Khatana, MD (Cincinnati, OH, USA); Michael S Korenfeld, MD (Washington, MO, USA); Christopher Lin, MD (Redding, CA, USA); Thomas K Mundorf, MD (Charlotte, NC, USA); George Nardin, MD (Kailua, HI, USA); Elizabeth D Sharpe, MD (Mount Pleasant, SC, USA); Sriram Sonty, MD (Calumet City, IL, USA); Michael E Tepedino, MD (High Point, NC, USA); Gail L Torkildsen, MD (Andover, MA, USA); Robert D Williams, MD (Louisville, KY, USA).

\section{Disclosure}

ER Craven has no proprietary interest in the study drug or its manufacturer. He serves as a paid consultant to Allergan, Inc. CC Liu, AL Batoosingh, RM Schiffman, and SM Whitcup are employees of Allergan, Inc.

\section{References}

1. van der Valk R, Webers CA, Schouten JS, Zeegers MP, Hendrikse F, Prins $\mathrm{MH}$. Intraocular pressure-lowering effects of all commonly used glaucoma drugs: a meta-analysis of randomized clinical trials. Ophthalmology. 2005;112:1177-1185.

2. McKee HD, Gupta MS, Ahad MA, Saldana M, Innes JR. First-choice treatment preferences for primary open-angle glaucoma in the United Kingdom. Eye. 2005;19:923-924.

3. Patel SC, Spaeth GL. Compliance in patients prescribed eyedrops for glaucoma. Ophthalmic Surg. 1995;26:233-236.

4. OlthoffCM, Schouten JS, van de Borne BW, Webers CA. Noncompliance with ocular hypotensive treatment in patients with glaucoma or ocular hypertension: an evidence-based review. Ophthalmology. 2005;112: 953-961.

5. Holló G. The side effects of the prostaglandin analogues. Expert Opin Drug Saf. 2007;6:45-52.

6. Astin M, Stjernschantz J, Selén G. Role of nitric oxide in PGF2 alpha-induced ocular hyperemia. Exp Eye Res. 1994;59:401-407.
7. Chen J, Dinh T, Woodward DF, et al. Bimatoprost: mechanism of ocular surface hyperemia associated with topical therapy. Cardiovasc Drug Rev. 2005;23:231-246.

8. Higginbotham EJ, Schuman JS, Goldberg I, et al; For Bimatoprost Study Groups 1 and 2. One-year, randomized study comparing bimatoprost and timolol in glaucoma and ocular hypertension. Arch Ophthalmol. 2002; 120:1286-1293.

9. Leal BC, Medeiros FA, Medeiros FW, Santo RM, Susanna R Jr. Conjunctival hyperemia associated with bimatoprost use: a histopathologic study. Am J Ophthalmol. 2004;138:310-313.

10. Sherwood M, Brandt J. Six-month comparison of bimatoprost once-daily and twice-daily with timolol twice-daily in patients with elevated intraocular pressure. Surv Ophthalmol. 2001;45:S361-S368.

11. Watson P, Stjernschantz J; Latanoprost Study Group. A six-month, randomized, double-masked study comparing latanoprost with timolol in open-angle glaucoma and ocular hypertension. Ophthalmology. 1996; 103:126-137.

12. Fellman RL, Sullivan EK, Ratliff M, et al; Travoprost Study Group. Comparison of travoprost $0.0015 \%$ and $0.004 \%$ with timolol $0.5 \%$ in patients with elevated intraocular pressure: a 6-month, masked, multicenter trial. Ophthalmology. 2002;109:998-1008.

13. Feldman RM. Conjunctival hyperemia and the use of topical prostaglandins in glaucoma and ocular hypertension. JOcul Pharmacol Ther. 2003;19:23-35.

14. Denis P, Lafuma A, Khoshnood B, Mimaud V, Berdeaux G. A meta-analysis of topical prostaglandin analogues intra-ocular pressure lowering in glaucoma therapy. Curr Med Res Opin. 2007;23: 601-608.

15. Aptel F, Cucherat M, Denis P. Efficacy and tolerability of prostaglandin analogs: a meta-analysis of randomized controlled clinical trials. J Glaucoma. 2008;17:667-673.

16. Abelson MB, Mroz M, Rosner SA, Dirks MS, Hirabayashi D. Multicenter, open-label evaluation of hyperemia associated with use of bimatoprost in adults with open-angle glaucoma or ocular hypertension. Adv Ther. 2003;20:1-13.

17. Laibovitz RA, VanDenburgh AM, Felix C, et al. Comparison of the ocular hypotensive lipid AGN 192024 with timolol: dosing, efficacy, and safety evaluation of a novel compound for glaucoma management. Arch Ophthalmol. 2001;119:994-1000.

18. Katz LJ, Cohen JS, Batoosingh AL, Felix C, Shu V, Schiffman RM. Twelve-month, randomized, controlled trial of bimatoprost $0.01 \%$, $0.0125 \%$, and $0.03 \%$ in patients with glaucoma or ocular hypertension. Am J Ophthalmol. 2010;149:661-671.

19. Gandolfi SA, Cimino L. Effect of bimatoprost on patients with primary open-angle glaucoma or ocular hypertension who are nonresponders to latanoprost. Ophthalmology. 2003;110:609-614.

20. Gandolfi S, Simmons ST, Sturm R, Chen K, VanDenburgh AM; Bimatoprost Study Group 3. Three-month comparison of bimatoprost and latanoprost in patients with glaucoma and ocular hypertension. $A d v$ Ther. 2001;18:110-121.

21. Brandt JD, VanDenburgh AM, Chen K, Whitcup SM; Bimatoprost Study Group. Comparison of once- or twice-daily bimatoprost with twice-daily timolol in patients with elevated IOP: a 3-month clinical trial. Ophthalmology. 2001;108:1023-1031.

22. Cantor LB, Hoop J, Morgan L, WuDunn D, Catoira Y; BimatoprostTravoprost Study Group. Intraocular pressure-lowering efficacy of bimatoprost $0.03 \%$ and travoprost $0.004 \%$ in patients with glaucoma or ocular hypertension. Br J Ophthalmol. 2006;90:1370-1373.

23. The AGIS Investigators. The Advanced Glaucoma Intervention Study (AGIS): 7. The relationship between control of intraocular pressure and visual field deterioration. Am J Ophthalmol. 2000;130:429-440.

24. Law SK, Song BJ, Fang E, Caprioli J. Feasibility and efficacy of a mass switch from latanoprost to bimatoprost in glaucoma patients in a prepaid Health Maintenance Organization. Ophthalmology. 2005;112: 2123-2130.

25. Stewart WC, Holmes KT, Johnson MA. Washout periods for brimonidine $0.2 \%$ and latanoprost $0.005 \%$. Am J Ophthalmol. 2001;131:798-789. 
26. McCarey B, Edelhauser H. In vivo corneal epithelial permeability following treatment with prostaglandin analogs [correction of analoges] with or without benzalkonium chloride. J Ocul Pharmacol Ther. 2007; 23:445-451.

27. Pisella PJ, Debbasch C, Hamard P, et al. Conjunctival proinflammatory and proapoptotic effects of latanoprost and preserved and unpreserved timolol: an ex vivo and in vitro study. Invest Ophthalmol Vis Sci. 2004; 45:1360-1368.

28. Guenoun JM, Baudouin C, Rat P, Pauly A, Warnet JM, BrignoleBaudouin F. In vitro study of inflammatory potential and toxicity profile of latanoprost, travoprost, and bimatoprost in conjunctiva-derived epithelial cells. Invest Ophthalmol Vis Sci. 2005;46:2444-2450.

29. Russ HH, Costa VP, Ferreira FM, et al. Conjunctival changes induced by prostaglandin analogues and timolol maleate: a histomorphometric study. Arq Bras Oftalmol. 2007;70:910-916.

30. Whitson JT, Cavanagh HD, Lakshman N, Petroll WM. Assessment of corneal epithelial integrity after acute exposure to ocular hypotensive agents preserved with and without benzalkonium chloride. Adv Ther. 2006;23:663-671.

31. Liang H, Baudouin C, Pauly A, Brignole-Baudouin F. Conjunctival and corneal reactions in rabbits following short- and repeated exposure to preservative-free tafluprost, commercially available latanoprost and 0.02\% benzalkonium chloride. Br J Ophthalmol. 2008;92:1275-1282.

32. Kahook MY, Noecker RJ. Comparison of corneal and conjunctival changes after dosing of travoprost preserved with sofZia, latanoprost with $0.02 \%$ benzalkonium chloride, and preservative-free artificial tears. Cornea. 2008;27:339-343.
33. Kahook MY, Noecker R. Quantitative analysis of conjunctival goblet cells after chronic application of topical drops. Adv Ther. 2008;25: 743-751.

34. Gross RL, Peace JH, Smith SE, et al. Duration of IOP reduction with travoprost BAK-free solution. J Glaucoma. 2008;17:217-222.

35. Lewis RA, Katz GJ, Weiss MJ, et al; Travoprost BAC-free Study Group. Travoprost $0.004 \%$ with and without benzalkonium chloride: a comparison of safety and efficacy. J Glaucoma. 2007;16:98-103.

36. Hamacher T, Airaksinen J, Saarela V, Liinamaa MJ, Richter U, Ropo A. Efficacy and safety levels of preserved and preservative-free tafluprost are equivalent in patients with glaucoma or ocular hypertension: results from a pharmacodynamics analysis. Acta Ophthalmol Suppl (Oxf). 2008;242:14-19.

37. Camras CB, Alm A, Watson P, Stjernschantz J; Latanoprost Study Groups. Latanoprost, a prostaglandin analog, for glaucoma therapy. Efficacy and safety after 1 year of treatment in 198 patients. Ophthalmology. 1996;103:1916-1924.

38. Goldberg I, Li XY, Selaru P, Paggiarino D. A 5-year, randomized, open-label safety study of latanoprost and usual care in patients with open-angle glaucoma or ocular hypertension. Eur J Ophthalmol. 2008; 18:408-416.

39. Kurtz S, Mann O. Incidence of hyperemia associated with bimatoprost treatment in naïve subjects and in subjects previously treated with latanoprost. Eur J Ophthalmol. 2009;19:400-403.
Clinical Ophthalmology

\section{Publish your work in this journal}

Clinical Ophthalmology is an international, peer-reviewed journal covering all subspecialties within ophthalmology. Key topics include: Optometry; Visual science; Pharmacology and drug therapy in eye diseases; Basic Sciences; Primary and Secondary eye care; Patient Safety and Quality of Care Improvements. This journal is indexed on

\section{Dovepress}

PubMed Central and CAS, and is the official journal of The Society of Clinical Ophthalmology (SCO). The manuscript management system is completely online and includes a very quick and fair peer-review system, which is all easy to use. Visit http://www.dovepress.com/ testimonials.php to read real quotes from published authors. 